\title{
STATISTICAL METHOD FOR DIMENSIONAL ANALYSIS OF MICRO/NANOPARTICLES DEPOSITED ONTO TEXTILE SUBSTRATES
}

\author{
LAURENȚIU -CHRISTIAN DINCĂ, IULIANA DUMITRESCU, MARIANA VAMEŞU \\ The National Research and Development Institute for Textiles and Leather (INCDTP), 16 \\ Lucretiu Patrascanu, 030508, Bucharest, Romania, laurentiu.dinca@certex.ro
}

\begin{abstract}
In this work it was developed a morphological analysis method of nano- and microparticles deposited on the textile substrate. This method is based on the statistical analysis of their dimensions measured by SEM (scanning electron microscopy). Towards this development, it was used a knit surface treated with $\mathrm{TiO}_{2}-\mathrm{N}-\mathrm{Fe}$ (treatment that gives photocatalytic, antibacterial and antifungal skills). The method consists in the processing of the SEM images (previously obtained) by the insertion of labels with dimension of nano/micro- particles and the calculation of statistical parameters of values obtained. It was processed 10 SEM images of the same material from different places investigated at same magnification (in this case $8000 \mathrm{X}$ ), each of these images being processed in software Scandium 5.0. In each image it was measured the dimension of $10 \mathrm{micro} /$ nano- particles. The requirement of a large number of dimensional measurements is because the size of the constituent particles in surface treatments, generally, has some variability. The major advantage of using Scandium 5.0 software is that it calculates automatically the statistical parameters of the set of measured values for all 10 images (average, minimum, maximum, number of values, standard deviation).
\end{abstract}

Keywords: SEM, statistic, dimension.

\section{INTRODUCTION}

Nanoparticles are particles with at least one dimension smaller than 1 micron and potentially as small as atomic and molecular length scales $(\sim 0.2 \mathrm{~nm})$. Nanoparticles can have amorphous or crystalline form and their surfaces can act as carriers for liquid droplets or gases. Examples of materials in crystalline nanoparticle form are metaloxide like $\mathrm{TiO}_{2}, \mathrm{CuO}, \mathrm{ZnO}, \mathrm{AgCl}, \mathrm{CuO}$. Nanoparticulate matter should be considered a distinct form of matter organisation, due to its distinct properties (large reactive surface area and quantum size effects). Many authors limit the size of nanomaterials to $50 \mathrm{~nm}$ or $100 \mathrm{~nm}$, the choice of this upper limit being justified by the fact that some physical properties of nanoparticles approach those of bulk when their size reaches these values. However, this size threshold varies with material type and cannot be the basis for such a classification. A legitimate definition extends this upper size limit to 1 micron (micrometer), the sub-micron range being classified as nano. Consequently, this mean the particles with dimensions higher or equal than 1 micrometer are named "microparticles".

We have developed a morphological analysis method of nano- and microparticles deposited on the textile substrate. This method is based on the statistical analysis of their dimensions measured by SEM (scanning electron microscopy).

In order to improve the dimensional accuracy of these measurements, simultaneously, the calibration of electron microscope was performed with a standard multiscale purchased. This calibration was necessary to guarantee the quality of dimensional measurements (lengths) at several orders of magnification. This standard, produced by Christine Gröpl, is intended for use in calibration of dimensional measurements for a wide range of magnification orders, being a silicon wafer lithographed with a network of lines located from each other at distances in range of $100 \div 2500 \mathrm{~nm}$. 
In this work it was used a knit surface treated with $\mathrm{TiO}_{2}-\mathrm{N}-\mathrm{Fe}$ (treatment that gives photocatalytic, antibacterial and antifungal skills).

This method consists in the processing of the SEM images (previously obtained) by the insertion of labels with dimension of nano/micro-particles and the calculation of statistical parameters of values obtained. This processing of SEM images was performed with a specialized software called Scandium 5.0.

\section{STATISTICAL METHOD}

Next, it will be described the statistical analysis method developed in this work, using as example the material marked 'T4 (a II-a tratare)', which is a fabric treated with micro/nano-particles of $\mathrm{TiO}_{2}-\mathrm{N}-\mathrm{Fe}$.

In figures $1 \div 10$ are shown the SEM images obtained at same magnification (8000X), which were obtained using scanning electron microscope FEI Quanta 200, before processing them:

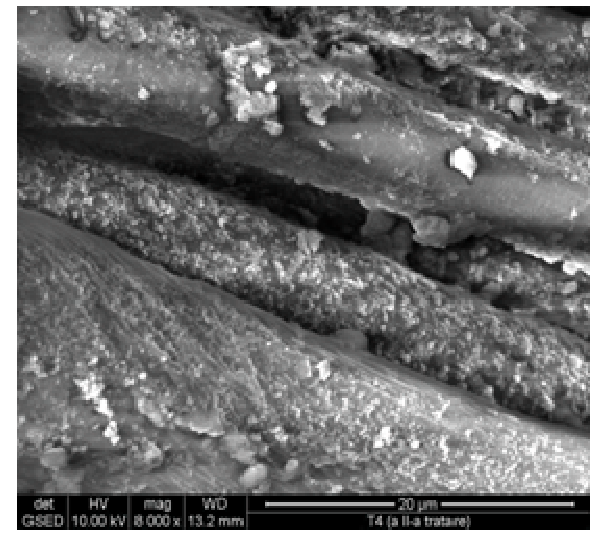

Figure 1. Unprocessed SEM image (no.1)

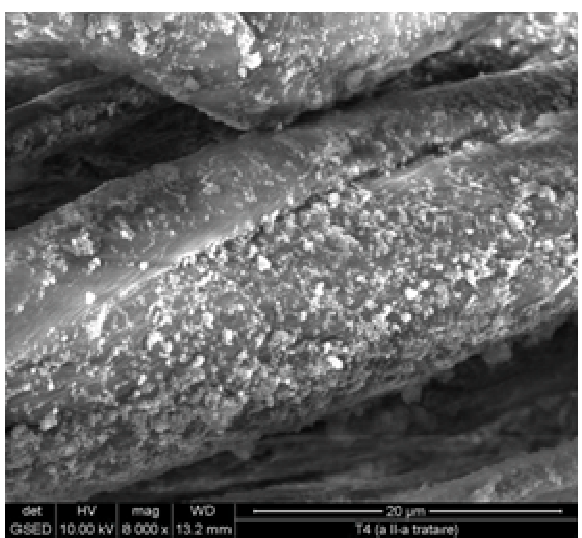

Figure 3. Unprocessed SEM image (no.3)

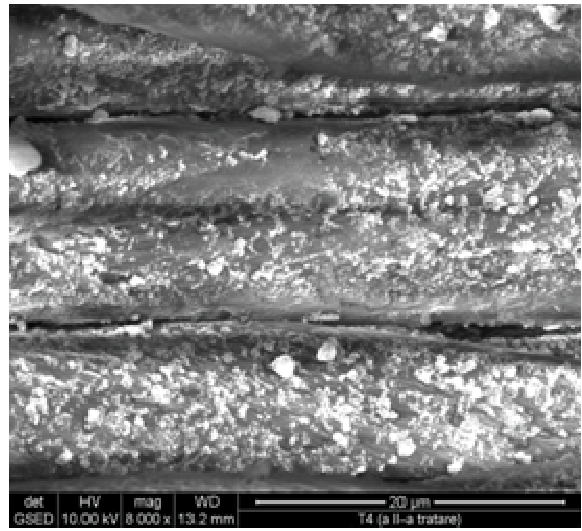

Figure 2. Unprocessed SEM image (no.2)

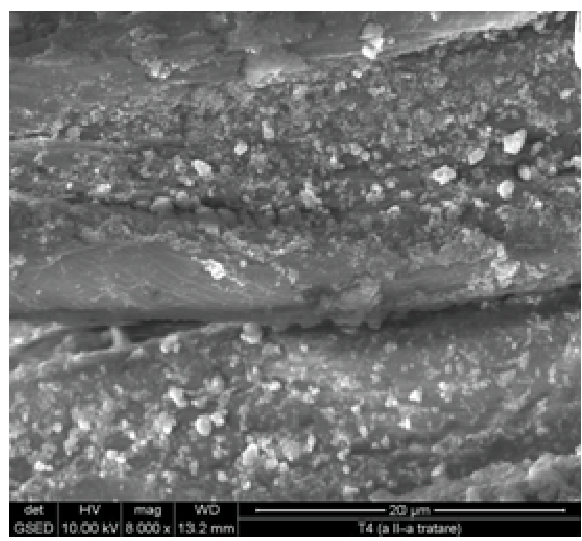

Figure 4. Unprocessed SEM image (no.4) 
ICAMS $2016-6^{\text {th }}$ International Conference on Advanced Materials and Systems

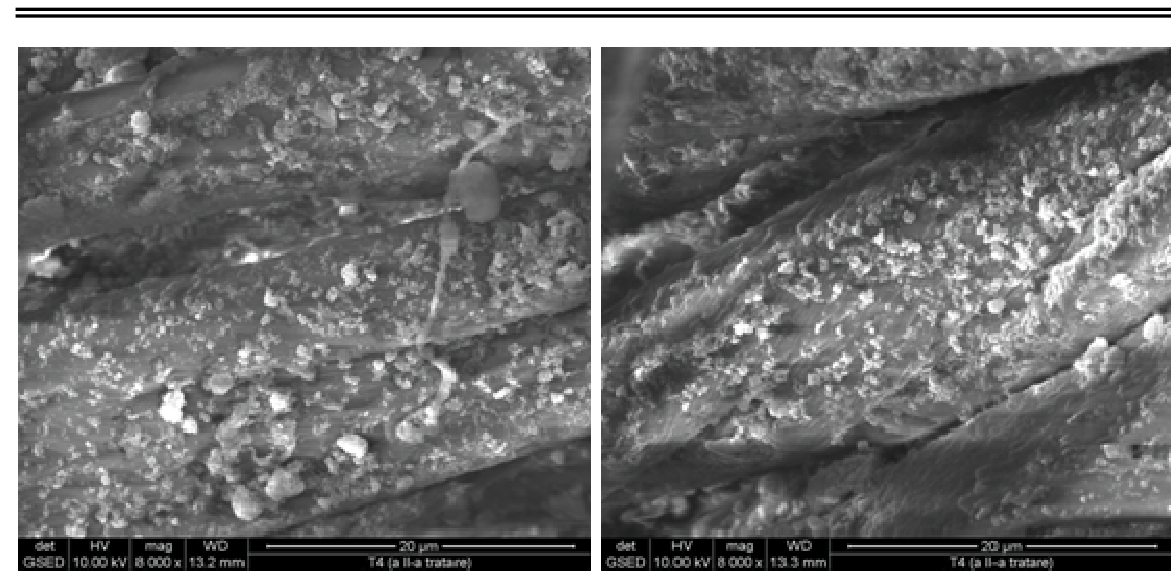

Figure 5. Unprocessed SEM image (no.5)

Figure 6. Unprocessed SEM image (no.6)
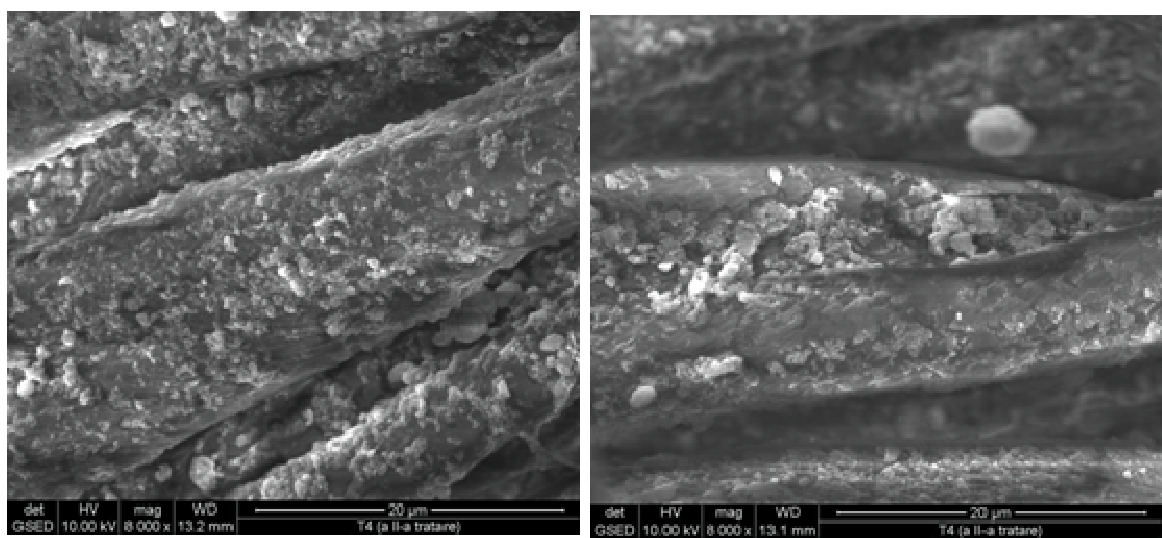

Figure 7. Unprocessed SEM image (no.7)

Figure 8. Unprocessed SEM image (no.8)
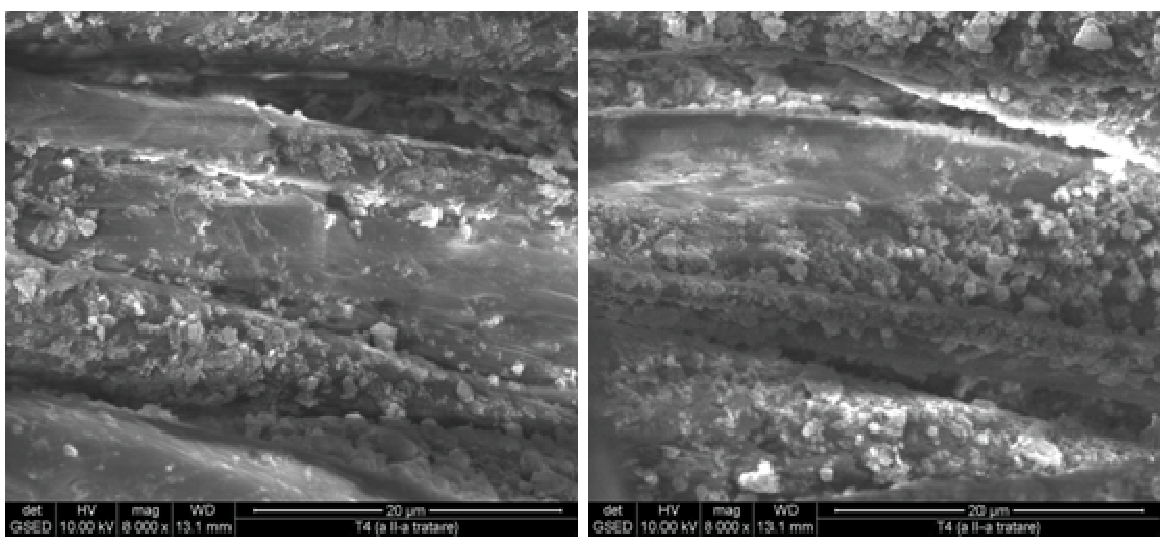

Figure 9. Unprocessed SEM image (no.9)

Figure 10. Unprocessed SEM image (no.10) 
After the purchase of 10 SEM images of the same material from different places investigated at same magnification (in this case $8000 \mathrm{X}$ ), each of these images is processed in software Scandium 5.0, measuring within each image the dimension of 10 micro/nano-particles.

The measure unit of particle size must be the same for all measurements of each image (in this case was chosen micrometer $-\mu \mathrm{m}$ ).

The requirement of a large number of dimensional measurements is because the size of the constituent particles in surface treatments, generally, have some variability. In this work were made 100 measurements (10 images x 10 measurements) onto the same textile material.

In figures $11 \div 20$ are shown the SEM images after processing in the software Scandium 5.0 (the screenshots of application window used are shown):

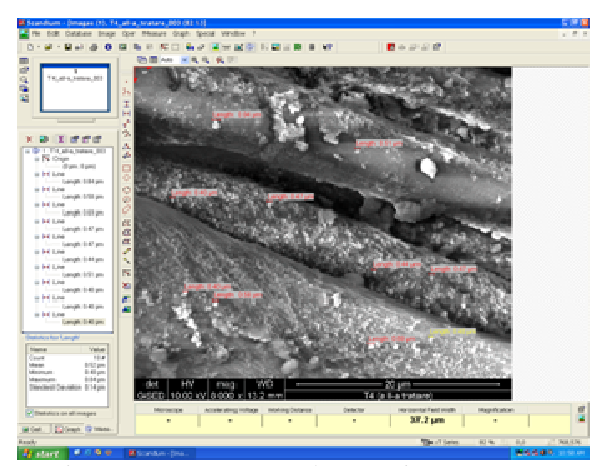

Figure 11. Processed SEM image (no.1)

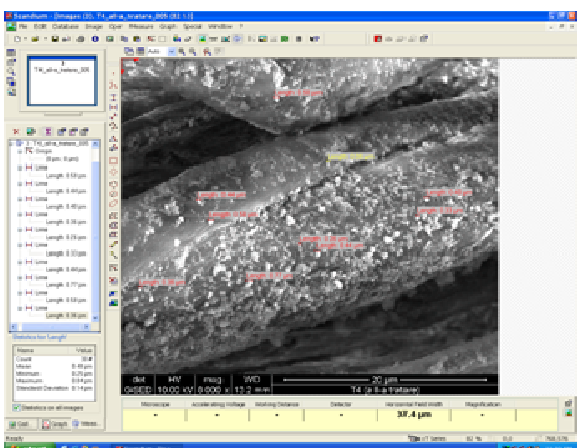

Figure 13. Processed SEM image (no.3)

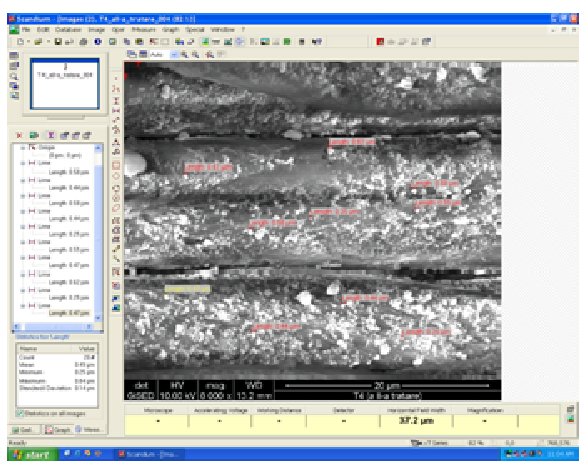

Figure 12. Processed SEM image (no.2)

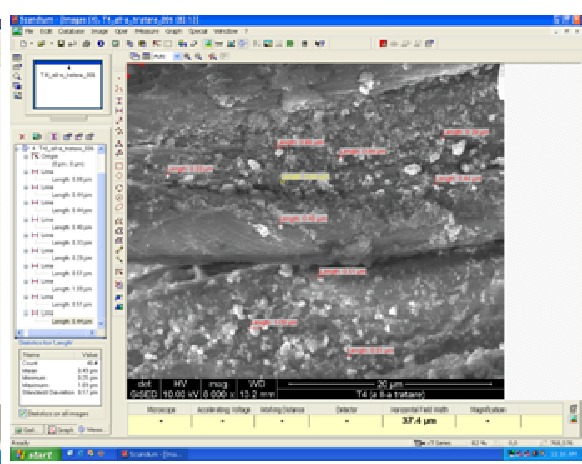

Figure 14. Processed SEM image (no.4) 
ICAMS $2016-6^{\text {th }}$ International Conference on Advanced Materials and Systems

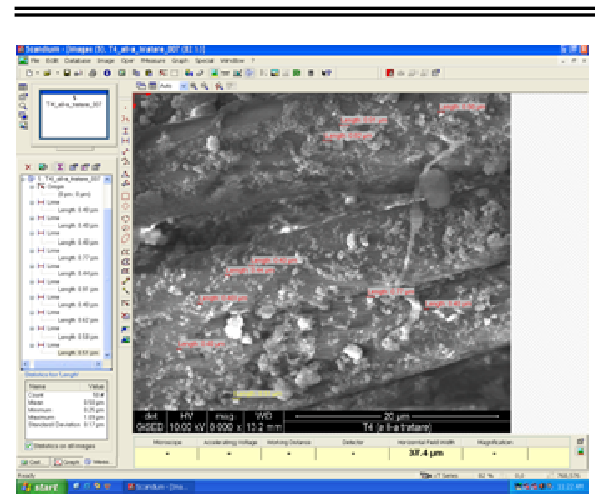

Figure 15. Processed SEM image (no.5)
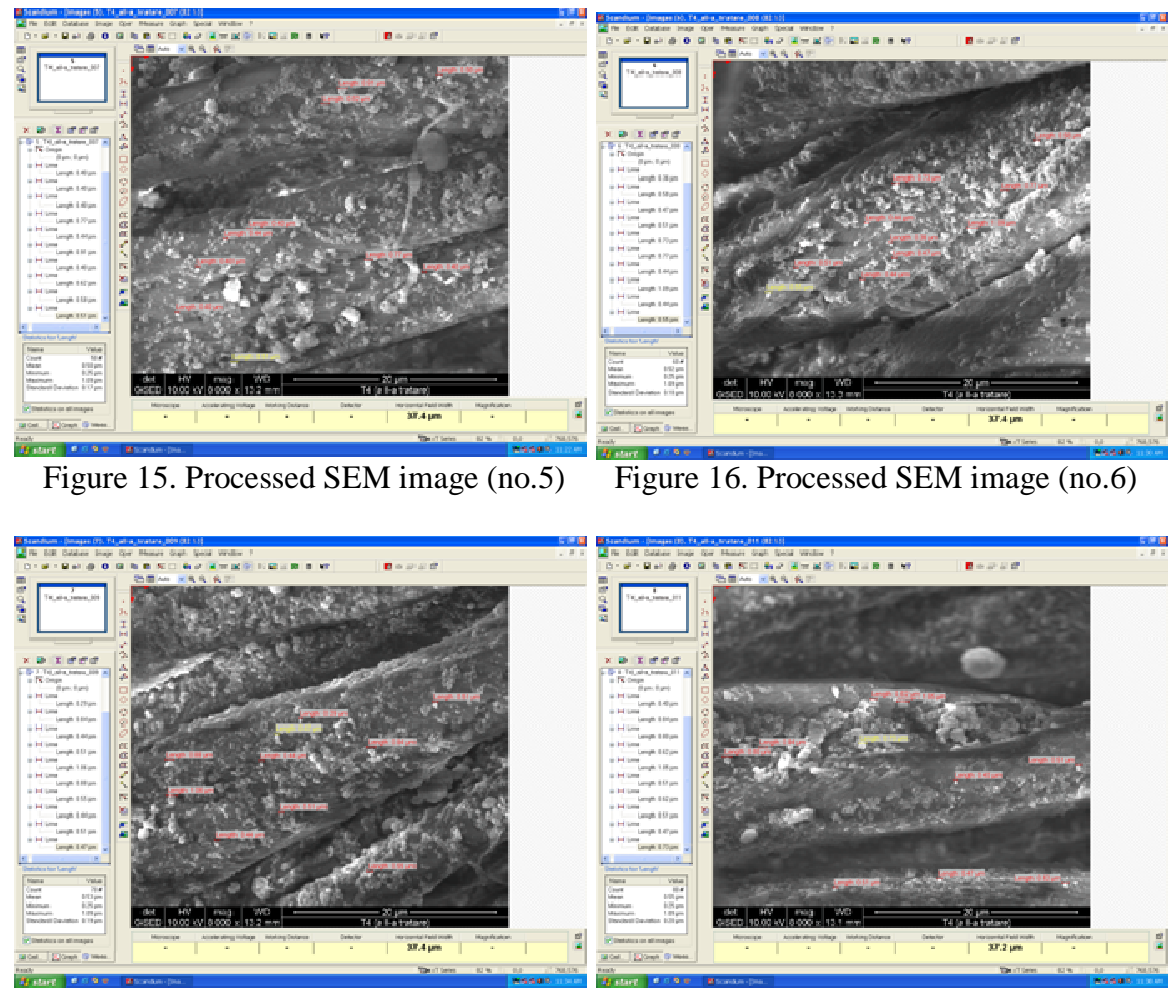

Figure 16. Processed SEM image (no.6)

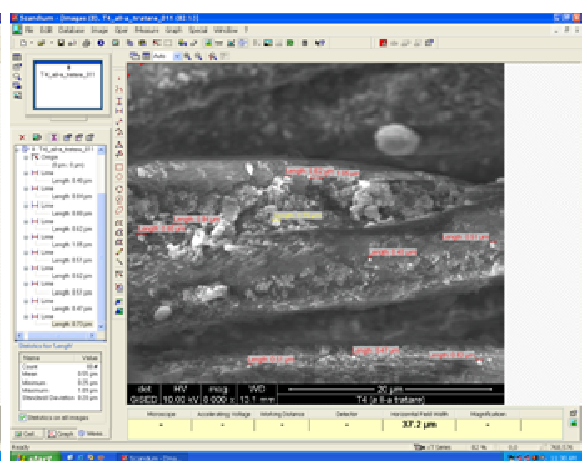

Figure 17. Processed SEM image (no.7)

Figure 18. Processed SEM image (no.8)
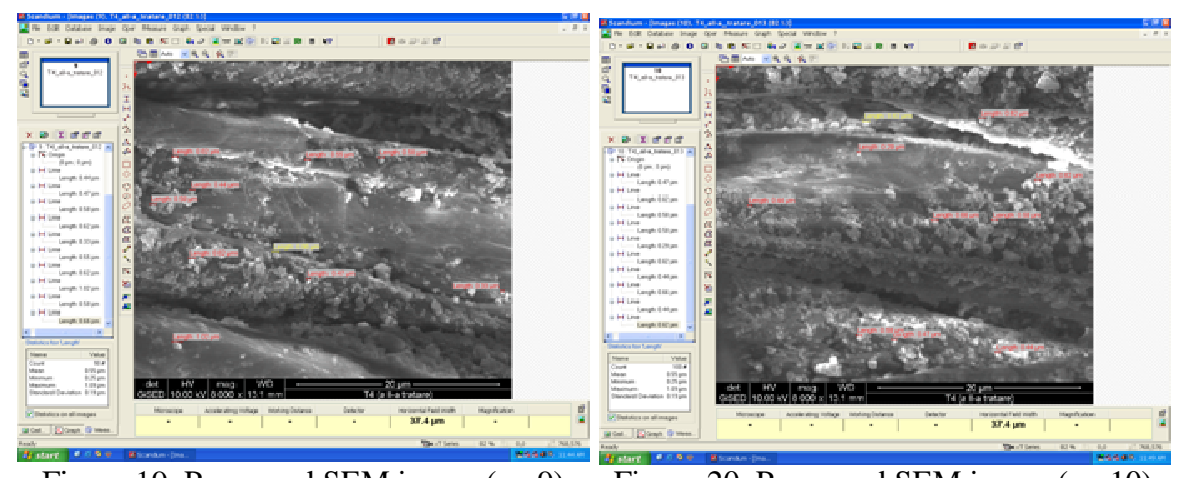

Figure 19. Processed SEM image (no.9)

Figure 20. Processed SEM image (no.10)

The major advantage of using Scandium 5.0 software is that it calculates automatically the statistical parameters of the set of measured values for all 10 images (average, minimum, maximum, number of values, standard deviation). The statistical parameters determined by the software are displayed in the bottom-left corner of the application window.

In this work, the software calculated for all 100 values of the particle size (dimension), the following values of the statistical parameters (Table 1): 
Statistical Method for Dimensional Analysis of Micro/Nanoparticles Deposited onto Textile Substrates

Table 1. Statistical parameters

\begin{tabular}{lc}
\hline Statistical parameter & $\begin{array}{c}\text { Value of statistical } \\
\text { parameter }\end{array}$ \\
\hline 1. Total number of measurement values & 100 \\
2. Average of measurement values & $0.55 \mu \mathrm{m}$ \\
3. Minimum of measurement values & $0.25 \mu \mathrm{m}$ \\
4. Maximum of measurement values & $1.09 \mu \mathrm{m}$ \\
5. Standard deviation of measurement values & $0.19 \mu \mathrm{m}$ \\
\hline
\end{tabular}

These values of statistical parameters listed in Table 1 (for the 100 measurement values) are displayed in the window which corresponds with tenth processed image (in the screenshot from Figure 20). The reason is that the software updates the values of statistical parameters at each measurement added in the current image imported into Scandium 5.0. For this reason, the final result of the statistical calculation for the whole set of measurements is displayed in the window that contains the latest SEM image imported into software.

As shown in Figures $11 \div 20$, dimensional measurement values are inserted in the SEM images imported. The operator is who chooses formations (particles) to which measured size. The formations (particles), which it is measured size, are chosen by the operator.

\section{CONCLUSIONS}

This statistical analysis of the dimension of particles from textile surface treatments, is useful to evaluate the grade of variability of particle sizes. Also, this method serves for categorizing the type of particles (nanoparticles or microparticles) from treatments investigated. In case of this work, by statistical point of view, the particles analysed may be considered nanoparticles (average and standard deviation of sizes is lower than $1 \mu \mathrm{m})$.

\section{Acknowledgements}

This paper was realized within Nucleus-Programme, performed with the support of The National Authority for Scientific Research and Innovation from Romania (ANCSI), project nr. 26N / March $14^{\text {th }} 2016$.

\section{REFERENCES}

FEI Company (2005), Quanta Training Course, FEI Company, Eindhoven (Netherlands).

Buzea, C., Pacheco, I.I., and Robbie, K. (2007), "Nanomaterials and nanoparticles: Sources and toxicity", Biointerphases, 2(4), MR17 - MR172. 